\title{
Three-dimensional water entry of a solid body: a computational study
}

\author{
Andrea L. Facci ${ }^{\mathrm{a}, *}$, Maurizio Porfiri ${ }^{\mathrm{b}}$, Stefano Ubertini ${ }^{\mathrm{a}}$ \\ ${ }^{a}$ School of Engineering, University of Tuscia, 01100 Viterbo, Italia. \\ ${ }^{b}$ Department of Mechanical and Aerospace Engineering, New York University Tandon School of Engineering, Brooklyn, NY, \\ 11201 USA.
}

\begin{abstract}
Marine vessels are continuously subject to impulsive loading from impact on the water surface. Understanding and quantifying the hydrodynamics generated by the three-dimensional (3D) water impact of a solid body is central to the design of resilient and performing vessels. Computational fluid dynamics (CFD) constitutes a viable tool for the study of water entry problems, which may overcome some of the drawbacks associated with semi-analytical and experimental methods. Here, we present a new computational study of the $3 \mathrm{D}$ water entry of a solid body with multiple curvatures. The method of finite volume is utilized to discretize incompressible Navier-Stokes equations in both air and water, and the method of volume of fluid is employed to describe the resulting free-surface multiphase flow. Computational results are validated against available experimental findings obtained using particle image velocimetry in terms of both the flow kinetics and kinematics. Specifically, we demonstrate the accuracy of our CFD solution in predicting the overall force experienced by the hull, the pile-up phenomenon, the velocity field in the water, the distribution of the hydrodynamic loading, and the energy transfer during the impact. Our approach is expected to aid in the validation of new semi-analytical solutions and to offer a viable means for conducting parametric studies and design optimization on marine vessels.

Keywords: Computational fluid dynamics, hull slamming, particle image velocimetry, pressure reconstruction, water entry
\end{abstract}

\section{Introduction}

Understanding the hydrodynamics associated with entry of solid bodies into the water surface is pivotal to the design of marine vessels (Abrate, 2013; Faltinsen, 1993; Korobkin, 1996) and aerospace structures (Seddon and Moatamedi, 2006). Just as hull slamming determines the hydrodynamic stability of the vessels during sailing and maneuvering, it influences their structural lifetime (Faltinsen et al., 2004; Hughes et al., 2013; McCue, 2012). Hull slamming results into complex, three-dimensional (3D) impulsive loading, whose

\footnotetext{
*Corresponding author. Tel.: +390761 357676.

Email addresses: andrea.facci@unitus.it (Andrea L. Facci), mporfiri@nyu.edu (Maurizio Porfiri), stefano.ubertini@unitus.it (Stefano Ubertini)
} 
spatial distribution and temporal evolution are both sensitive to the geometry of the vessels and their operating speed (Abrate, 2013; Faltinsen, 1993; Faltinsen et al., 2004; Hughes et al., 2013; Korobkin, 1996; McCue, 2012; Seddon and Moatamedi, 2006). Notwithstanding the central role of the hull geometry, most of the technical literature has focused on two-dimensional (2D) water entry problems (Abrate, 2013).

In the realm of $2 \mathrm{D}$ approximations, mathematically-tractable potential flow solutions have been established to elucidate the hydrodynamics in water entry problems. Building on the seminal work by Wagner on the water entry of solid bodies with low deadrise angle (Wagner, 1932), significant progress has been made toward dissecting the role of large deadrise angles (Korobkin, 2004; Mei et al., 1999), geometric asymmetries (Judge et al., 2004; Korobkin and Malenica, 2005; Semenov and Iafrati, 2006), and hydroelastic phenomena (Khabakhpasheva and Korobkin, 2013; Panciroli and Porfiri, 2014; Shams and Porfiri, 2015). These efforts have contributed an improved understanding of water impact, isolating the key geometric and physical factors that shape the unsteady fluid-structure interaction. For example, these studies have clarified the mechanics underpinning the formation of a water pile-up in the vicinity of the impacting solid and helped determine the wetted length of the body as a function of its geometry.

These theoretical investigations have been supported by a number of computational models, which have contributed a refined dataset upon which analytical or semi-analytical solutions could be tested and offered further insight into the physics of the impact (Abrate, 2013; Hughes et al., 2013). Several computational schemes have been proposed to study 2D water entry problems, ranging from traditional computational fluid dynamics (CFD) solvers (Colicchio et al., 2006; Das and Batra, 2011; Facci et al., 2015; Luo et al., 2011; Maki et al., 2011; Stenius et al., 2006; Sun and Faltinsen, 2006; Wang et al., 2012; Wang and Soares, 2013a,b) to emerging discrete fluid dynamics methods (De Rosis et al., 2014; Gong et al., 2009; Oger et al., 2006; Panciroli et al., 2012; Shao, 2009; Yang et al., 2012; Zarghami et al., 2014). Different from theoretical treatments based on Wagner solution, computational schemes enable a precise quantification of viscous phenomena along with a refined analysis of the dynamics of the water surface, including the pile-up phenomenon. Their implementation can be easily adjusted to closely simulate experimental conditions, with respect to both the real geometry of the hull and the setup.

In this paper, we seek to advance the field of computational modeling through the study of 3D hydrodynamics generated by the impact of a solid body on the water surface. We consider the complex geometry proposed in (Jalalisendi et al., 2015a) to proxy the slamming of a ship hull in a laboratory experimental setting. Specifically, we model the free fall of a solid body whose geometric curvature varies with respect to both its length and width. Our computational scheme builds on our prior work (Facci et al., 2015), where we have established an accurate and versatile CFD toolbox to study the 2D impact of a wedge. Here, we extend such a computational scheme to enable the analysis of $3 \mathrm{D}$ water entry, toward a detailed analysis of the pile-up phenomenon, a precise quantification of the hydrodynamic loading experienced by the body on its wetted surface, and a rigorous assessment of the energy transfer during water entry. This extension is 
motivated by the experimental results in Jalalisendi et al. (2015a), which offer compelling evidence for the importance of 3D phenomena, not captured by 2D approximations, in the impact of ship hulls models with multiple curvatures. Our approach is based on the finite volume method (Patankar, 1980) to discretize the incompressible Navier-Stokes equations in air and water, and the volume of fluid technique to describe the resulting multiphase flow (Rider and Kothe, 1998; Rusche, 2003; Scardovelli and Zaleski, 1999). Different from the finite element analysis presented in (Wang and Soares, 2014), our framework utilizes an open source software and focuses on a complex hull geometry, with multiple curvatures.

We demonstrate the accuracy of our computational approach through comparison with experimental data from (Jalalisendi et al., 2015a), where particle image velocimetry (PIV) is used to measure the 3D flow physics generated by the impact and reconstruct the associated pressure field. PIV is a classical technique in experimental fluid dynamics which uses images of a seeded flow to non-intrusively measure the velocity in the fluid from the motion of the seeding tracers (Raffel et al., 1998). By scanning the flow field in 2D through planar PIV, the methodology proposed in (Jalalisendi et al., 2015a,b) affords the systematic measurement of the three components of the velocity vector everywhere in the water. From the knowledge of the $3 \mathrm{D}$ velocity field and the identification of both the solid body and the free surface, Navier-Stokes equations can be numerically integrated to estimate the pressure field by extending the approach proposed in (Nila et al., 2013; Panciroli and Porfiri, 2013, 2015; Panciroli et al., 2015; Shams et al., 2015) from 2D to 3D.

Contrasting computational findings with PIV results, we provide a thorough validation of our numerical solution, which can aid in the development of new semi-analytical approaches and in the unprecedented quantification of $3 \mathrm{D}$ effects. With respect to the use of pressure gauges, accelerometers, and load cells which have been pervasive in experimental studies on water entry (Carcaterra and Ciappi, 2004; Luo et al., 2012; Peterson et al., 1997; Tveitnes et al., 2008; Van Nuffel et al., 2013; Van Nuffel et al., 2014; Wu et al., 2004), PIV allows for verifying numerical predictions on both the pressure and velocity everywhere in the water, which would otherwise be not feasible (Wang and Soares, 2014). This knowledge is in turn utilized to quantify the spatial distribution and temporal evolution of the loading experienced by the hull and estimate the energy transfer during the impact. Our experimentally-validated computational approach can be effectively used as a valid alternative to more costly and labor-intensive experimental schemes in parametric studies and design optimization.

The rest of the paper is organized as follows. In Section 2, we briefly describe the problem under investigation. In Section 3, we summarize the experimental scheme presented in (Jalalisendi et al., 2015a) and detail our computational approach. In Section 4, we present our main results, which include the prediction of the overall force experienced by the hull, the pile-up dynamics, the water velocity, the hydrodynamic loading, and the energy transfer. Section 5 contains the conclusions of our study and an outlook on future work. 


\section{Problem statement}

(a) Longitudinal section

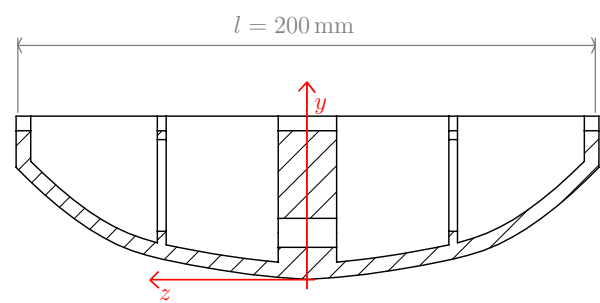

(c) Plant view

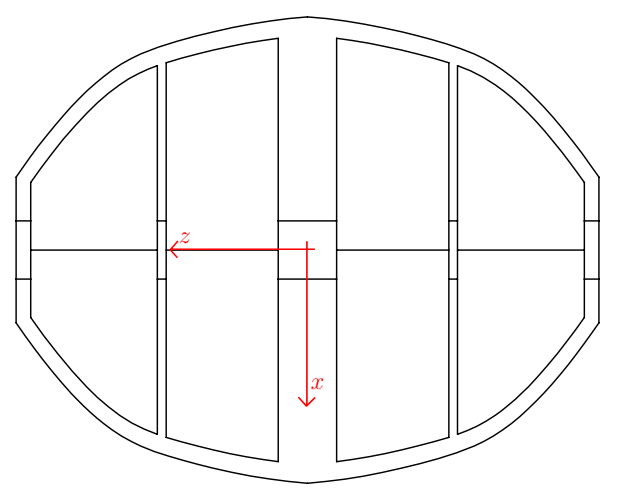

(b) Axonometry

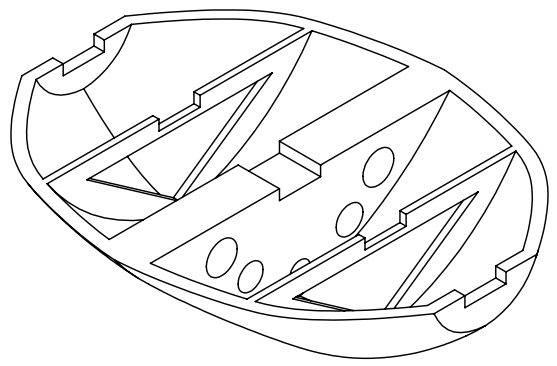

(d) Front view

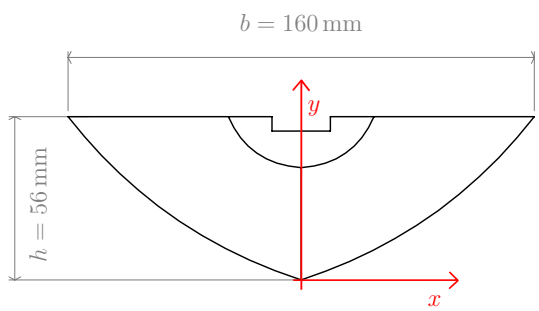

Figure 1: Schematic representation of the impacting hull: (a) longitudinal section, (b) axonometry, (c) plant view, and (d) front view. The hull position in the fixed reference frame refers to the time of the impact.

Table 1: Geometrical and physical properties of the specimen utilized for experiments and simulations. Data from Jalalisendi et al. (2015a).

\begin{tabular}{llrl}
\hline \hline Property & Symbol & Value & Units \\
\hline Length & $l$ & 200 & $\mathrm{~mm}$ \\
Width & $b$ & 160 & $\mathrm{~mm}$ \\
Height & $h$ & 56 & $\mathrm{~mm}$ \\
Mass & $M$ & 0.601 & $\mathrm{~kg}$ \\
Drop height & $\xi_{0}$ & 500 & $\mathrm{~mm}$ \\
\hline \hline
\end{tabular}

The geometry of the specimen is sketched in Figure 1. Relevant properties, such as the length $l$, width dimensions (The exact shape of the hull can be found in (Jalalisendi et al., 2015a) ${ }^{1}$ ). The specimen, is

\footnotetext{
${ }^{1}$ One of the coefficients was erroneously presented in Table 1: $a_{4}=219.912$ instead of $a_{4}=-219.912$.
} 
constrained to move only along the vertical direction using a system of rails, and it is released from a height of $\xi_{0}=500 \mathrm{~mm}$ above the quiescent water surface. The impact takes place at time $t=0$ with a velocity of $3.1 \mathrm{~m} / \mathrm{s}$.

A fixed Cartesian reference frame is placed with the $y$-axis along the vertical direction, and the $z$-axis along the specimen length. The origin of the reference frame is at the intersection of the specimen symmetry planes and the undisturbed water surface.

The maximum velocity of the specimen is much lower compared to the speed of sound in air and water, and we consider the two fluids, air and water, immiscible. Thus, the flow in our computational model is described through the mass and momentum conservation equations

$$
\begin{aligned}
\nabla \cdot \boldsymbol{u} & =0, \\
\frac{\mathrm{D}(\rho \boldsymbol{u})}{\mathrm{D} t} & =-\nabla p+\nabla \cdot \mu \nabla \boldsymbol{u}+\rho \boldsymbol{g} .
\end{aligned}
$$

Here $\boldsymbol{u}$ is the fluid (air or water) velocity; $p$ is the pressure; $\mu$ is the dynamic viscosity; $\rho$ is the density; $\boldsymbol{g}=-g \boldsymbol{j}$ is the gravitational acceleration, with $g$ being its magnitude and $j$ the unit vector along the $y$-axis; and $\mathrm{D}(\cdot) / \mathrm{D} t$ represents the material derivative. Since the flow is multiphase, $\rho$ and $\mu$ vary in space and time according to the local fluid state of aggregation, that is, gas or liquid, or, equivalently, air or water. In particular, the air density and viscosity are $\rho_{\mathrm{a}}=1 \mathrm{~kg} / \mathrm{m}^{3}$ and $\mu_{\mathrm{a}}=1.48 \times 10^{-5}$ Pas respectively, and the water density and viscosity are $\rho_{\mathrm{w}}=1000 \mathrm{~kg} / \mathrm{m}^{3}$ and $\mu_{\mathrm{w}}=1 \times 10^{-3} \mathrm{Pas}$, respectively.

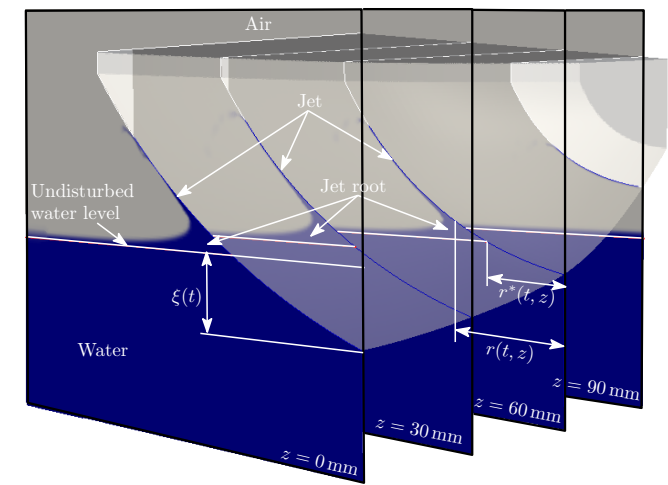

Figure 2: Definition of relevant symbols for the water impact.

Figure 2 reports the principal features of the flow generated by the impact of the hull on the water surface, including: the penetration $\xi(t)$ of the specimen with respect to the undisturbed water level $(\xi(0)=0)$; the reference wetted width $r^{*}(t, z)$, corresponding to the intersection of the specimen profile with the undisturbed water level; and the wetted width $r(t, z)$, estimated from the location of the jet root. As a consequence of the pile-up phenomenon, $r$ is generally different than $r^{*}$, and we define the pile-up coefficient as (Abrate, 
2013; Facci et al., 2015)

$$
\phi=\frac{r}{r^{*}} .
$$

The water impact generates a hydrodynamic force on the solid body, which can be computed from the stress in the fluid as,

$$
\boldsymbol{F}=\int_{\Sigma}\left\{-p \boldsymbol{I}+\mu\left[\nabla \boldsymbol{u}-\nabla \boldsymbol{u}^{\mathrm{T}}\right]\right\} \boldsymbol{n} \mathrm{d} \Sigma,
$$

where $\boldsymbol{I}$ is the identity tensor, $\Sigma$ is the body surface, and $\boldsymbol{n}$ is the unit normal to the outer surface of the specimen. The mechanical energy in a generic portion of the fluid domain $\Omega$ is

$$
E=\int_{\Omega}\left(\frac{1}{2} \rho|\boldsymbol{u}|^{2}+\rho g y\right) \mathrm{d} \Omega .
$$

\section{Methods}

\subsection{Experimental setup}

The complete details of the experimental setup can be found in (Jalalisendi et al., 2015a). The experimental apparatus is comprised of an aluminum frame that holds a $800 \times 320 \times 350 \mathrm{~mm}^{3}$ water tank. The frame has two $1.5 \mathrm{~m}$ guiding rails standing on top of the water tank. To constrain the movement of the specimen along the vertical direction, the specimen is rigidly attached to a sledge that runs along the rails during free fall, before vertically impacting the water free surface, see Figure 3.

The data acquisition system is composed of a time-resolved planar PIV system utilized to measure the 2D velocity field in multiple cross-sections of the specimen. The PIV system consists of two high speed cameras and a laser source. The water inside the tank is seeded with silver-coated hollow glass spheres with a mean diameter of $44 \mu \mathrm{m}$. The laser sheet from the laser source illuminates the particles on the measurement plane and the two high speed cameras are used to record the fluid flow generated by the impact at an acquisition frequency of $4 \mathrm{kHz}$ (Jalalisendi et al., 2015a), yielding a resolution of $4.99 \mathrm{pixel} / \mathrm{mm}$. The cameras are synchronized and positioned at an angle of $117^{\circ}$ with respect to the impact plane; this angle is chosen to reduce aberrations at the air-water surface due to the 3D free-surface elevation (Jalalisendi et al., 2015a).

To reconstruct the 3D velocity field from planar PIV data, we perform two sets of PIV experiments. In the first set, images are recorded for fixed values of $z$ along the length of the body, called cross-sectional PIV, while in the second set, images are acquired the experiments are conducted for fixed values of $x$ along the width, called axial PIV. Due to symmetry, PIV experiments are conducted on a quarter of the specimen. A total of 11 equally spaced locations are considered for cross-sectional PIV starting from the mid-span $(z=0)$ to the end of the specimen $(z=100 \mathrm{~mm})$, while in axial PIV, 9 equally spaced locations are chosen starting from the keel $(x=0)$ to the edge $(x=90 \mathrm{~mm})$. The step size for both cross-sectional and axial $\mathrm{PIV}$ is $10 \mathrm{~mm}$. 


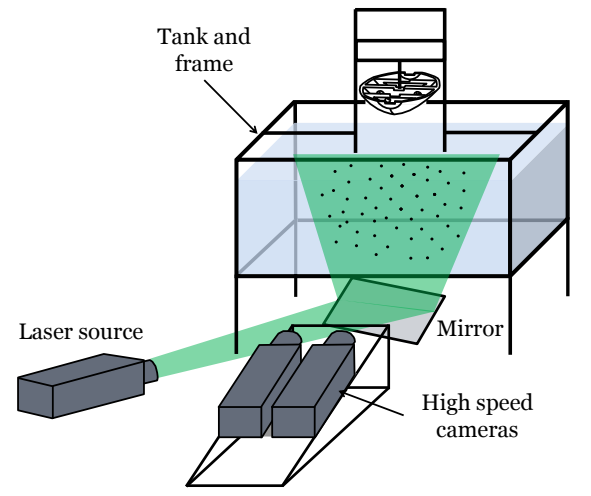

Figure 3: Schematics of the experimental apparatus and the data acquisition system.

To accurately measure the entry depth and acceleration of the falling body, an additional set of experiments is conducted using a single camera and mounting an accelerometer on top of the sledge. The entry depth is estimated by tracking the displacement of markers drawn on the sledge, while the acceleration is directly measured from the accelerometer.

PIV analyses are performed on the set of acquired images using the open source MATLAB graphical user interface "PIVlab" (Thielicke and Stamhuis, 2014a,b). The complete details of the PIV analysis can be found in (Jalalisendi et al., 2015a). PIV analysis provides a sequence of equally spaced velocity grid for each experiment. To identify the wedge surface and water free surface in each experiment, we utilize a dedicated image masking procedure (Jalalisendi et al., 2015b; Panciroli and Porfiri, 2013). The masking data are also used to estimate the time traces of the wetted length and width, corresponding to the last wetted point on the body surface in each PIV measurement plane. These data are in turn utilized to synchronize data from cross-sectional and axial PIV. The time history of the wetted length from axial PIV at the keel is utilized to determine the time instant at which the water wets the specimen in each cross-sectional PIV plane. Similarly, the time history of the wetted length from cross-sectional PIV at the mid-span is used to identify the time instant at which the water wets the specimen in each axial PIV plane.

The procedure for fusing cross-sectional and axial PIV toward the reconstruction of the 3D velocity is detailed in (Jalalisendi et al., 2015a), building on the technique previously introduced in (Jalalisendi et al., 2015b). The axial PIV dataset provides the axial velocity component $w$ as a function of $y$ and $z$, and it is used to enrich each cross-sectional PIV grid, where the components $u$ and $v$ in the $x y$-planes are available, with an estimation of the axial flow. Specifically, for each frame in cross-sectional PIV, we identify overlapping velocity grid points between that plane and axial PIV planes to perform the enrichment. Axial velocity data for the remaining grid points are estimated using a cubic spline interpolation scheme (Jalalisendi et al., 2015a). 


\subsection{PIV-based pressure reconstruction}

The pressure field on each cross-sectional PIV plane is reconstructed only inside the water, similarly to the approach used in (Jalalisendi et al., 2015b). We neglect the effect of viscosity in Eq. (1b) and perform the reconstruction for each value of $z$ (Jalalisendi et al., 2015a) by using 2D incompressible Navier-Stokes equations

$$
\begin{aligned}
& \frac{\partial p}{\partial x}=-\rho_{w}\left(\frac{\partial u}{\partial t}+u \frac{\partial u}{\partial x}+v \frac{\partial u}{\partial y}+w \frac{\partial u}{\partial z}\right) \\
& \frac{\partial p}{\partial y}=-\rho_{w}\left(\frac{\partial v}{\partial t}+u \frac{\partial v}{\partial x}+v \frac{\partial v}{\partial y}+w \frac{\partial v}{\partial z}\right)-\rho_{w} g .
\end{aligned}
$$

The pressure field at each frame is calculated by first setting the pressure to zero at the outermost point of the free surface; calculating the pressure along the outer boundary of the liquid except the body surface, the water free surface and the symmetry boundary, utilizing the forward integration technique used by Panciroli and Porfiri (2013); and finally, computing the pressure field on the entire liquid using the approach proposed by Baur and Köngeter (1999).

The total vertical force exerted on the specimen is calculated by integrating the pressure values on the wetted surface. Specifically, the vertical force as a function of time is

$$
F_{y}=\int_{\Sigma}-p(\boldsymbol{n} \cdot \boldsymbol{j}) \mathrm{d} \Sigma,
$$

where $p$ is the pressure on the wetted surface, and $\boldsymbol{n}$ is the unit normal to the body surface. To calculate the integral in Eq. (6) we use trapezoidal rule. Different from Eq. (3), the experimental evaluation of the impact force uses only the surface which is wetted by the water, since PIV data are not available in air.

\subsection{PIV-based energy computation}

With reference to Figure 4 , we define $\Omega_{\text {bulk }}$ as the quarter of the volume of the liquid moved by the impacting body excluding the risen water, comprising the pile-up and the spray jet. PIV is able to only capture a portion of the liquid bulk, shown as the red dashed box in Figure 4. We partition the water region captured by PIV into $\Omega_{1}$ and $\Omega_{2}$, representing the liquid bulk captured by PIV analysis and the risen water, respectively. The risen water consists of the volume of the liquid in the pile-up and the spray jet. The energy in the bulk $\Omega_{\text {bulk }}$ can be written as

$$
\begin{aligned}
E_{\mathrm{bulk}} & =\int_{\Omega_{1}}\left(\frac{1}{2} \rho_{w}|\boldsymbol{u}|^{2}+\rho_{w} g y\right) \mathrm{d} \Omega_{1}+ \\
& +\int_{0}^{t} \int_{\Gamma_{1} \cup \Gamma_{2} \cup \Gamma_{3}}\left(\frac{1}{2}|\boldsymbol{u}|^{2}+g y+\frac{p}{\rho_{w}}\right) \\
& \rho_{w}(\boldsymbol{u} \cdot \boldsymbol{n}) \mathrm{d} \Gamma .
\end{aligned}
$$


The first term is the part of the energy transferred to the bulk that is captured by our PIV analysis. In particular, we calculate the energy per unit length transferred to the fluid bulk at each measurement location $z$ and then integrate along the length of the body. The second term is the energy transferred from $\Omega_{1}$ to the rest of the bulk through $\Gamma_{1}, \Gamma_{2}$, and $\Gamma_{3}$ (with unit normal $\boldsymbol{n}$ ). The integrals on the surfaces $\Gamma_{1}, \Gamma_{2}$, and $\Gamma_{3}$ are calculated by integrating the energy rate per unit length leaving $\Omega_{1}$ over the length of the body. All the integrals are computed using the trapezoidal rule.

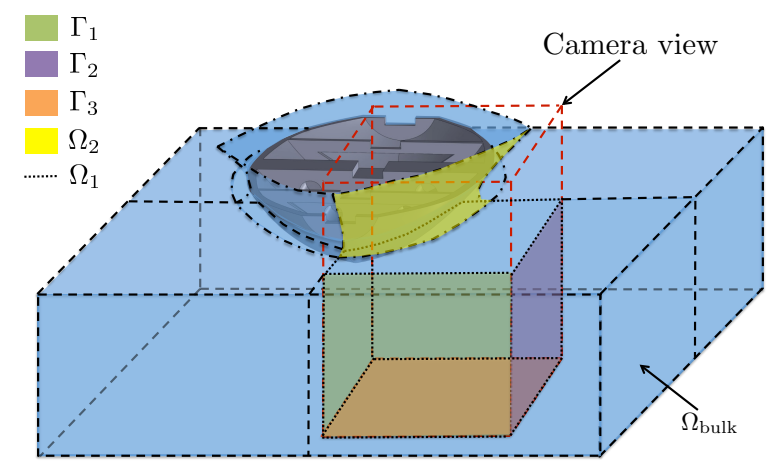

Figure 4: Schematics of the control volume analysis with overlaid notations. The red box indicates the quarter of the problem captured during PIV experiments.

\subsection{Numerical model}

The 3D system of differential equations in Eq. (1) is numerically integrated through the interDyMFoam solver of the OpenFOAM-2.4 (open source) software package (OpenFOAM, 2015). This solver uses the finite volume method (Patankar, 1980) to discretize equations (1a) and (1b), and the volume of fluid (VOF) technique to model the multiphase flow (Facci et al., 2015; Rider and Kothe, 1998; Rusche, 2003; Scardovelli and Zaleski, 1999). The VOF describes the free surface evolution by numerically integrating the following equation (Rusche, 2003)

$$
\frac{\mathrm{D} \alpha}{\mathrm{D} t}+\nabla \cdot\left[\boldsymbol{U}_{r} \alpha(1-\alpha)\right]=0
$$

where $\alpha$ is the water volume fraction and $\boldsymbol{U}_{r}$ is the relative velocity of the two phases. The second term of Eq. (8) is introduced to compensate the numerical smearing of the fluid interface (Rusche, 2003).

Temporal discretization, is performed through the first order accurate Euler implicit scheme (OpenFOAM Foundation, 2013). The Gauss linear-upwind scheme is utilized discretize the convective terms in Eq. (1), and the Gauss Van Leer scheme is employed for the convective term of Eq. (8). Other spatial discretizations are performed through the Gauss linear procedure. The discretized Navier-Stokes equations are solved through the non iterative pressure implicit with split operators (PISO) algorithm (Issa, 1985), while the 


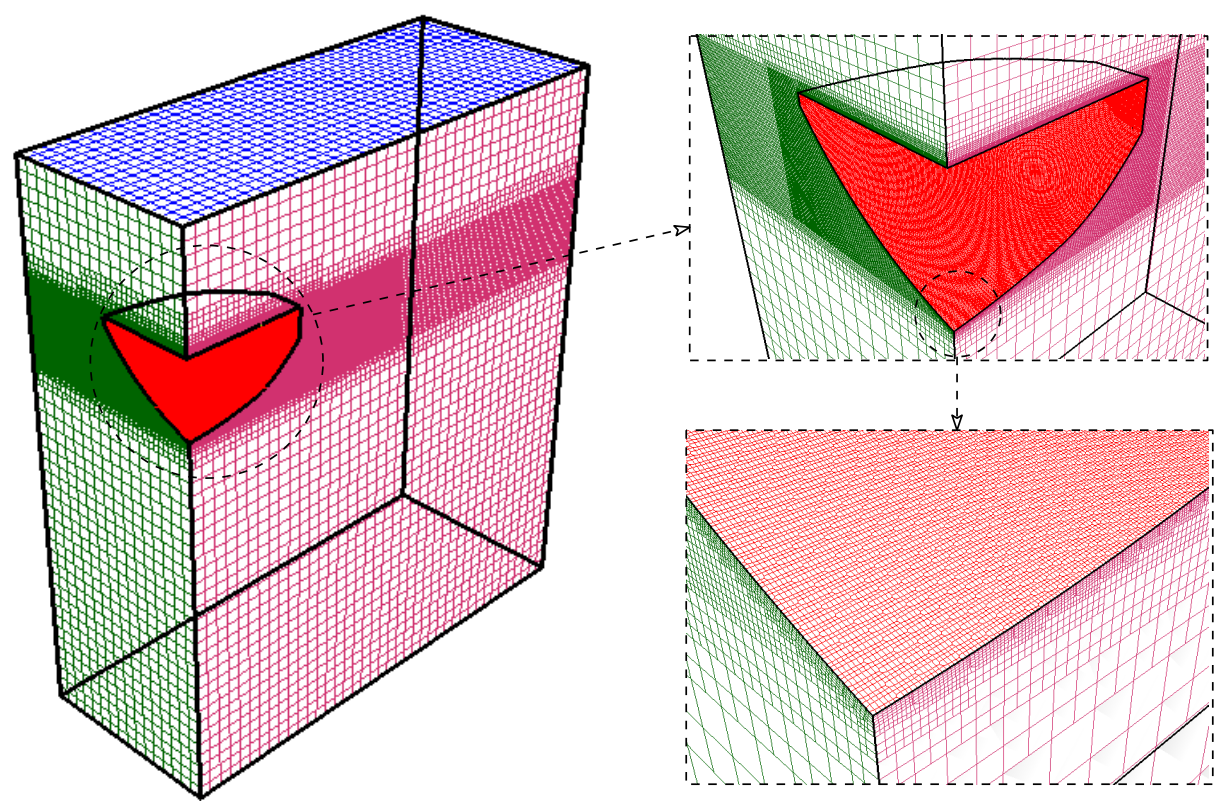

Figure 5: Illustration of the computational mesh in the vicinity of the impacting hull.

discretized version of Eq. (8) is solved using the multi-dimensional universal limiter with explicit solution (MULES) procedure (OpenFOAM Foundation, 2013). All the resulting linear systems are solved through the geometric agglomerated algebraic multi grid iterative algorithm (OpenFOAM Foundation, 2013).

The computational domain is discretized using the hexa-dominant unstructured mesh shown in Figure 5 which is obtained through the shappyHexMesh pre-processor (OpenFOAM Foundation, 2013). The maximum linear cell dimension is $10 \mathrm{~mm}$ and the minimum one is $3.13 \times 10^{-1} \mathrm{~mm}$, resulting in approximately $4.2 \times 10^{6}$ cells. Only one quarter of the physical domain is simulated due to the symmetry of the specimen with respect to the $x y$-plane and to the $y z$-plane.

The boundary conditions applied at the domain borders are: (i) no-slip and $\boldsymbol{n} \cdot \nabla p=0$ on the hull surface; (ii) symmetry on $y z$-plane and $x y$-plane; (iii) $p=0$ and $\boldsymbol{n} \cdot \nabla \boldsymbol{u}=0$ on the top boundary; and (iv) free-slip and $\boldsymbol{n} \cdot \nabla p=0$ on the bottom and right walls. Some remarks on the accuracy of these boundary conditions for a body falling in free fall can be found in (Facci et al., 2015).

Since this paper concentrates on the analysis of the 3D hydrodynamics ensuing from the water entry of a complex 3D body, we opt for constraining the specimen to translate along the vertical direction following the experimental data in Jalalisendi et al. (2015a). This methodological choice affords the systematic validation of computational results on the flow physics, without potential confounds associated with mismatches in the motion of the body between experimental and computational datasets. The experimental displacement is interpolated using a third order polynomial, which, is differentiated in time to obtain the velocity $\dot{\xi}$. The fitted time traces for $\xi(t)$ and $\dot{\xi}(t)$, shown in Figure 6 , are used as input to the numerical model. 
(a) Depth

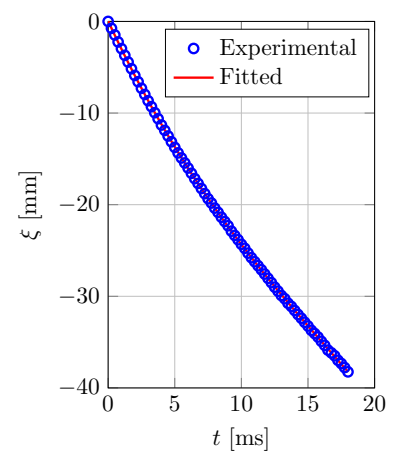

(b) Velocity

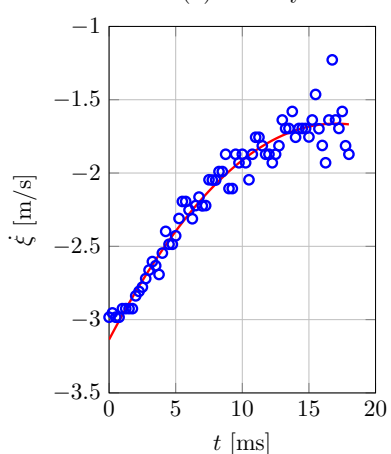

Figure 6: (a) Entry depth and (b) velocity measured during the impact, along with fitted data used as input to the numerical model. Experimental velocity data are computed using a first order Euler approximation of the entry depth.

Equation (3) is numerically evaluated with the following second order accurate formulation:

$$
\boldsymbol{F}=\sum_{i=1}^{N_{\text {hull }}}\left\{-p_{i} \boldsymbol{I}+\mu_{i}\left[(\nabla \boldsymbol{u})_{i}-(\nabla \boldsymbol{u})_{i}^{\mathrm{T}}\right]\right\} \boldsymbol{n}_{i}\left|\Sigma_{i}\right|,
$$

where $N_{\text {hull }}$ is the number of cells on the specimen surface, $\left|\Sigma_{i}\right|$ is the area of a generic surface element, $\boldsymbol{n}_{i}$ is the unit vector normal to the surface element, and $\mu_{i}=\alpha_{i} \mu_{\mathrm{w}}+\left(1-\alpha_{i}\right) \mu_{\mathrm{a}}$ is the dynamic viscosity of the fluid in each surface element.

We calculate the mechanical energy in the fluid by integrating Eq. (4), as

$$
E=\sum_{j=1}^{N}\left[\frac{1}{2} \rho_{j}\left|\boldsymbol{u}_{j}\right|^{2} \Omega_{j}+\rho_{j} g y_{j}\left|\Omega_{j}\right|\right],
$$

where $N$ is the number of computational cells in the fluid domain, $\left|\Omega_{j}\right|$ is the volume of the $j$-th cell, $y_{j}$ is the position of its center of gravity along the $y$-axis, and $\rho_{j}=\alpha_{j} \rho_{\mathrm{w}}+\left(1-\alpha_{j}\right) \rho_{\mathrm{a}}$ is the density of the fluid in the $j$-th cell.

To reduce the computational time, simulations are performed using the OpenFOAM parallel computing capabilities. The domain is decomposed into 12 sub-domains and about 24 hours are required to complete a simulation utilizing Intel(R) Xeon(R) CPU E5-2687W v2 processors.

\section{Results and discussion}

\subsection{Hydrodynamic force}

Figure 7 reports the vertical hydrodynamic force, $F_{y}$, acting on the hull measured using the accelerometer together with numerical result from Eq. (9). CFD accurately captures the rise of the force in time and the time at which the hydrodynamic loading is maximized. Numerical results tend to slightly overestimate the 
(a) Force

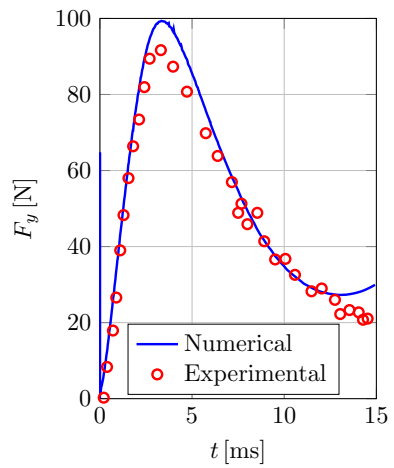

(b) Relative error in the force

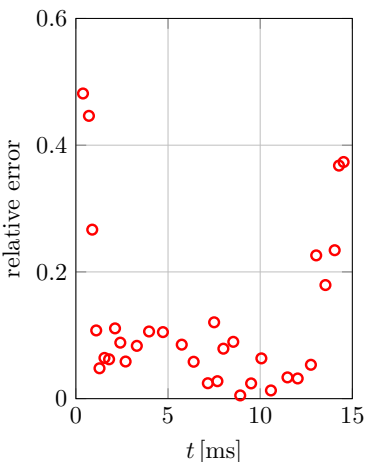

Figure 7: Vertical component of the hydrodynamic force, $F_{y}$, on the impacting specimen: comparison between numerical and experimental results. (a) Force and (b) relative error, computed as the absolute value of the difference between numerical and experimental results divided by the experimental value.

(a) $z=1 \mathrm{~mm}$

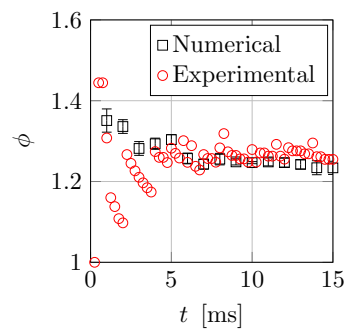

(b) $z=30 \mathrm{~mm}$

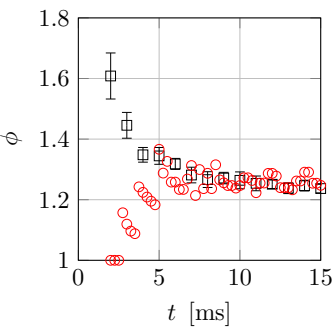

(c) $z=60 \mathrm{~mm}$

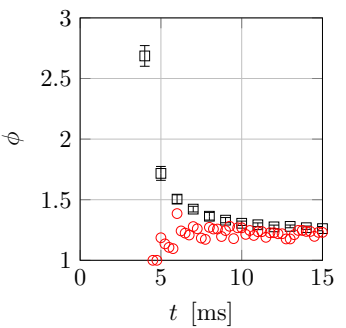

(d) $z=90 \mathrm{~mm}$

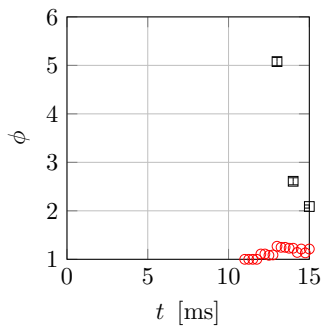

Figure 8: Pile-up coefficient as a function of time for different $x y$-planes: (a) $z=1$ [mm], (b) $z=30[\mathrm{~mm}],(\mathrm{c}) z=60[\mathrm{~mm}]$, and (d) $z=90[\mathrm{~mm}]$. Error bars are estimated on the basis of the CFD cell size.

maximum force of about $8 \%$. The largest difference between the vertical force evaluated through CFD and indirectly measured using the accelerometer is approximately $8.5 \mathrm{~N}$.

Figure 7(b) shows that the relative error is maximized in the very early stage of the impact, for $t<1 \mathrm{~ms}$, when the CFD is significantly influenced by the initial conditions imposed to the computational model, and in the later stage of the impact, for $t>12 \mathrm{~ms}$ where a precise estimation of the specimen motion is hampered by the large scatter on the velocity, as evidenced in Figure 6(b). During most of the impact, the relative error is below 15\%. Such a discrepancy is in line with the inherent uncertainties of the experiment, for instance in the precise evaluation of the specimen motion.

\subsection{Pile-up dynamics}

The evolution of the free-surface position during the impact is of great interest for the study of water entry phenomena. To measure the extent of the pile-up phenomenon in Figure 8 we show numerical predictions against experimental findings for the pile-up coefficient. For all the specimen sections, both CFD and experiments settle down to $\phi \simeq 1.25$ for a sufficiently large time after the onset of the impact. This value, 


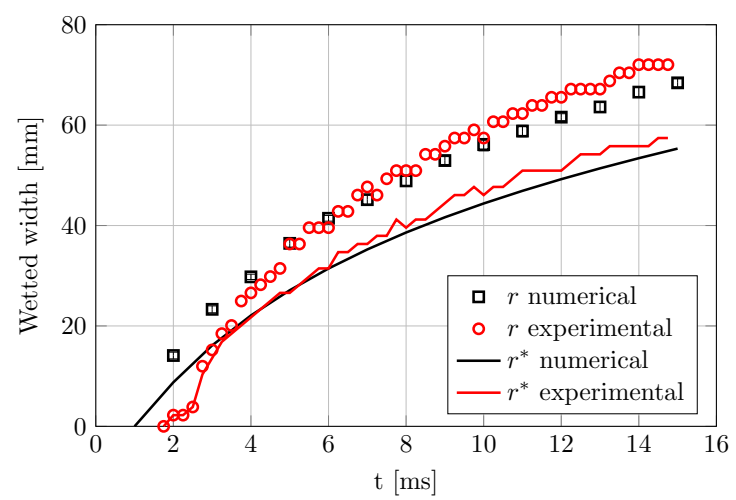

Figure 9: Wetted width as a function of the time after the onset of the impact for $z=30 \mathrm{~mm}$. Note that the reference wetted width is theoretically evaluated in CFD from the prescribed entry depth and the hull geometry, while it is experimentally determined for PIV.

(a) $t=2 \mathrm{~ms}$

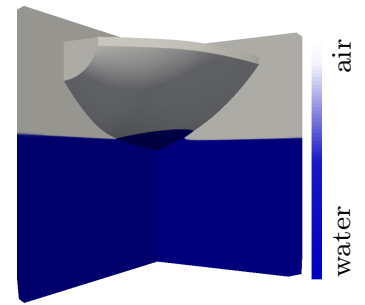

(d) $t=9 \mathrm{~ms}$

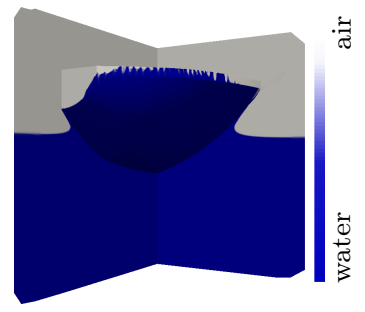

(b) $t=4 \mathrm{~ms}$

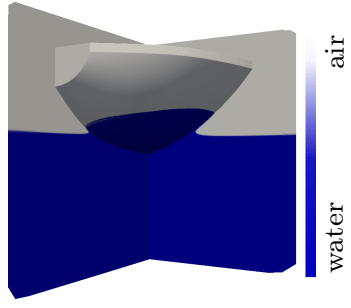

(e) $t=12 \mathrm{~ms}$

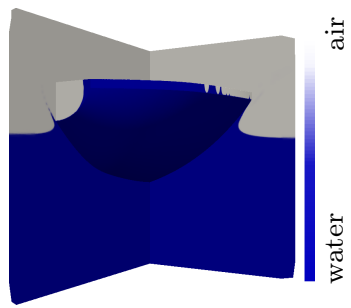

(c) $t=6 \mathrm{~ms}$

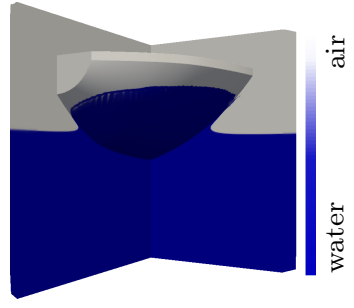

(f) $t=15 \mathrm{~ms}$

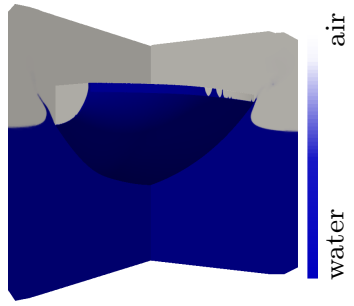

Figure 10: Water level contour plots on the hull and on the two symmetry planes at different time instants: (a) $t=2 \mathrm{~ms}$, (b) $t=4 \mathrm{~ms}$, (c) $t=6 \mathrm{~ms}$, (d) $t=9 \mathrm{ms,} \mathrm{(e)} t=12 \mathrm{~ms}$, and (f) $t=15 \mathrm{~ms}$.

as also discussed in (Jalalisendi et al., 2015a) for 3D experiments and (Facci et al., 2015) in 2D simulations, is lower than the value of $\pi / 2$, theoretically hypothesized (Wagner, 1932) for the $2 \mathrm{D}$ steady-state impact of a wedge.

For the first $3 \mathrm{~ms}$ after each cross-section has entered the water surface, numerical findings and experimental results are not in agreement. The computational analysis predicts an initially large value of $\phi$, which then gradually decreases as the penetration depth increases. On the contrary, experimental results indicate that $\phi$ is initially one and progressively grows toward the steady state. The evaluation of the pile-up 
(a) $t=2 \mathrm{~ms}$

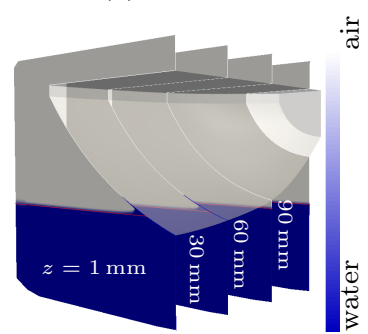

(d) $t=9 \mathrm{~ms}$

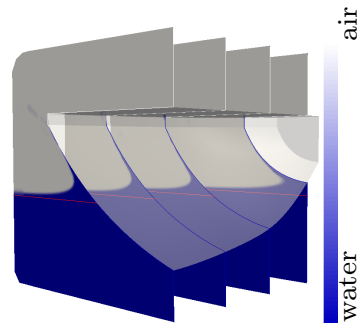

(b) $t=4 \mathrm{~ms}$

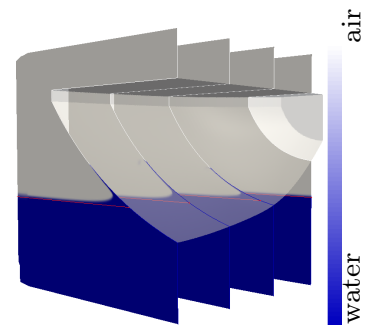

(e) $t=12 \mathrm{~ms}$

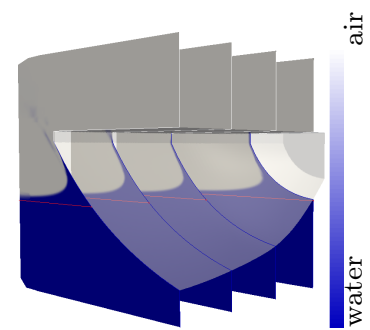

(c) $t=6 \mathrm{~ms}$

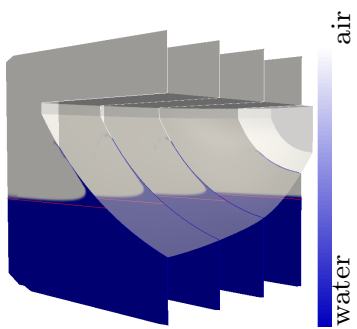

(f) $t=15 \mathrm{~ms}$

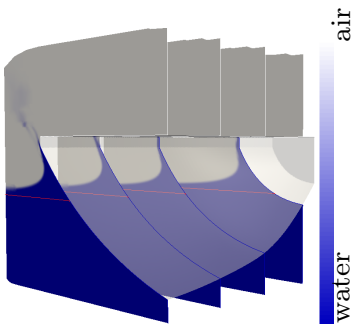

Figure 11: Water level contour plots on different $x y$-planes and at different time instants: (a) $t=2 \mathrm{~ms}$, (b) $t=4 \mathrm{~ms}$, (c) $t=6 \mathrm{~ms}$, (d) $t=9 \mathrm{~ms}$, (e) $t=12 \mathrm{~ms}$, and (f) $t=15 \mathrm{~ms}$. The red lines identify the undisturbed water surface.

coefficient during the very early stage of the impact is particularly challenging in both the numerical study and the experiments, due to the limited amount of risen water. In fact, during the first 3 ms of the impact, the wetted width and its reference value are on the order of few millimeters, which is comparable with the order of magnitude of the CFD cell size, varying between $0.1 \mathrm{~mm}$ and $10 \mathrm{~mm}$, and PIV resolution which only affords few pixels per millimeter, as shown in Figure 9.

The pile-up dynamics can be further elucidated by analyzing the time evolution of the liquid volume fraction $\alpha$ for different times and cross-sections. Figure 10 illustrates the effect of the 3D curvature of the hull on the pile-up dynamics by showing $\alpha$ on the impacting body and on the $x y$ - and $y z$ - planes. In both these planes, we note the formation of a pile-up region where the water rises above the undisturbed free surface level, creating a thin jet flow which detaches from the body. The pile-up phenomenon differs in the two symmetry planes, due to the different curvature of the hull, which influences the fluid-structure interaction. This is also evident from the uneven elevation of of the wetted surface of the hull with respect to the $y$-axis. Figure 11 further details the dependence of the pile-up dynamics on the hull shape. Each cross-section enters the water at a different time due to the curvature in the $y z$-plane. In addition, the prior impact of cross-sections with smaller values of $z$ influences the free-surface elevation, whereby only the cross-section at $z=0$ enters a flat free surface. As a result, a cross-section may be wetted even though its nominal wetted length, computed on the basis of the $x z$-plane, is zero. This offers an indirect explanation for the numerical trend of $\phi$ in the early stage of the impact, as shown in Figure 8. Neglecting 3D phenomena 
(a) $t=2 \mathrm{~ms}$

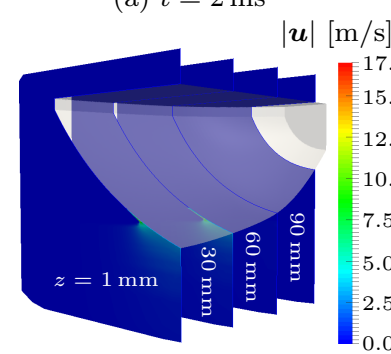

(d) $t=9 \mathrm{~ms}$

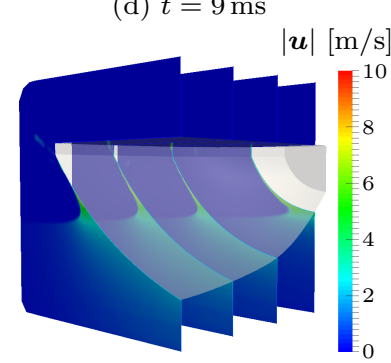

(b) $t=4 \mathrm{~ms}$ $|\boldsymbol{u}|[\mathrm{m} / \mathrm{s}]$

(c) $t=6 \mathrm{~ms}$ $|\boldsymbol{u}|[\mathrm{m} / \mathrm{s}]$

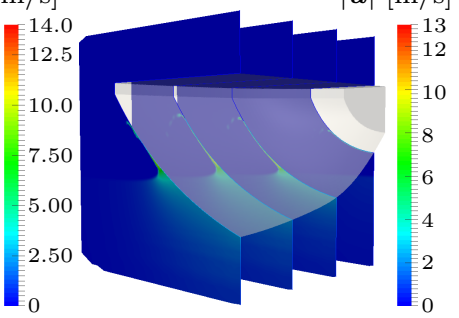

(e) $t=12 \mathrm{~ms}$

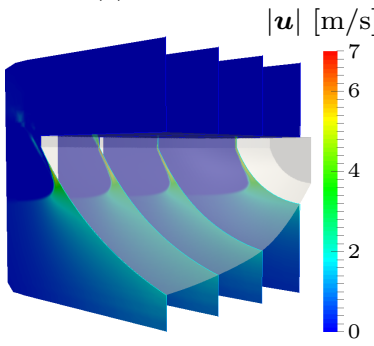

Figure 12: Velocity magnitude contour plots on different $x y$-planes and at different time instants: (a) $t=2 \mathrm{~ms}$, (b) $t=4 \mathrm{~ms}$, (c) $t=6 \mathrm{~ms}$, (d) $t=9 \mathrm{~ms}$, (e) $t=12 \mathrm{~ms}$, (f) $t=15 \mathrm{~ms}$. Only the water velocity is represented for clarity, and the velocity of the air is set to zero.

Figure 12 shows the velocity magnitude obtained through CFD at different cross-sections and at different times. To highlight the effect of the water, in Figure 12 we display only the velocity field within the water domain, and we represent the entire air volume as $|\boldsymbol{u}|=0$. The large velocity magnitude attained in the air cushion between the free surface and the impacting hull would have hindered any consideration on the water hydrodynamics. A comparison of Figure 11 to Figure 12 demonstrates that the water velocity is consistently maximized in the risen water, and in particular in the spray region, in agreement with experimental results in (Facci et al., 2015; Jalalisendi et al., 2015a,b), and gradually decreases towards the keel where $|\boldsymbol{u}| \simeq \dot{\xi}$. The water velocity reaches the global maximum immediately after the impact, when the specimen velocity is larger and the portion of the specimen with the lower local deadrise angle is impacting the free surface.

The axial component of the water velocity, displayed in Figure 13, is a distinctive feature of 3D water entry, and its analysis helps isolate the effect of 3D phenomena on the hydrodynamic loading experienced by the solid body during water entry. Due to the problem symmetry, $w=0$ at $z=0$ where $\boldsymbol{u}$ lies on the $x y$-plane. The axial component of the velocity gradually increases along the length of the specimen, as $3 \mathrm{D}$ effects become relevant. The overall variation of the axial velocity is determined by the border effects and the curvature of the hull in the $y z$-plane. The latter is evidenced by comparing Figure 13 to Figure 10 , 
(a) $t=2 \mathrm{~ms}$

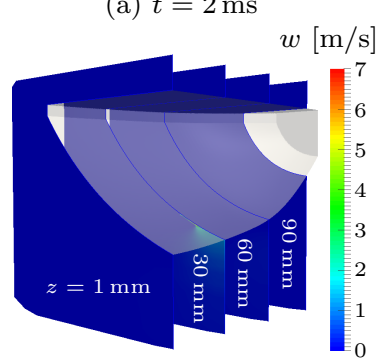

(d) $t=9 \mathrm{~ms}$

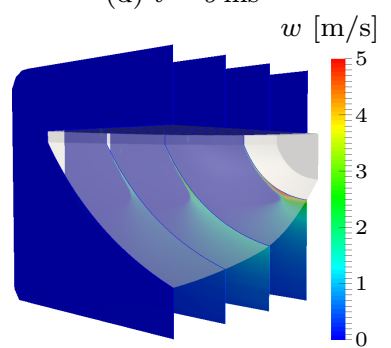

(b) $t=4 \mathrm{~ms}$

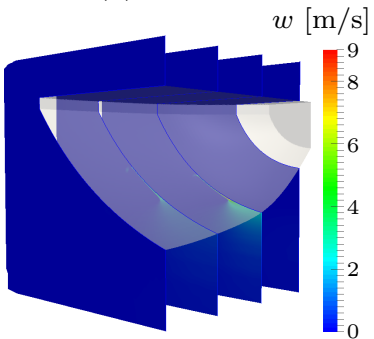

(e) $t=12 \mathrm{~ms}$ $w[\mathrm{~m} / \mathrm{s}]$

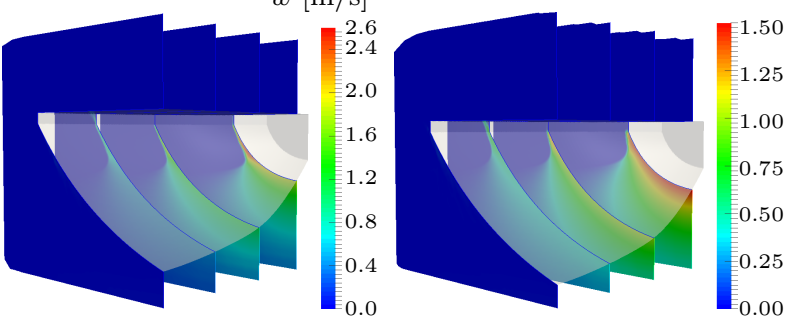

Figure 13: Axial velocity $w$ contour plots on different $x y$-planes and at different time instants: (a) $t=2 \mathrm{~ms},(\mathrm{~b}) t=4 \mathrm{~ms}$, (c) $t=6 \mathrm{~ms}$, (d) $t=9 \mathrm{~ms}$, (e) $t=12 \mathrm{~ms}$, (f) $t=15 \mathrm{~ms}$. Only the water velocity is represented for clarity, and the velocity of the air is set to zero.

especially for $t \leq 6 \mathrm{~ms}$, whereby we observe a marked increase of $w$ towards the outermost wetted portion of the specimen and in the risen water. When the specimen is completely submerged, the maximum value of $w$ is located at the bow, see Figure 13(f), as a consequence of both the hull curvature and edge effects, and large values of $w$ are attained also at the keel.

\subsection{Hydrodynamic pressure}

Figure 14 compares the pressure profile, obtained through CFD and PIV-based pressure reconstruction, on different cross-sections of the hull and at different times after the impact. Numerical and experimental results are presented only for $t \geq 6 \mathrm{~ms}$, when PIV-based pressure reconstruction should be considered accurate. As explained in (Facci et al., 2015; Panciroli and Porfiri, 2013), only a limited number of velocity vectors is initially available to PIV for pressure, thereby limiting the accuracy of the indirect pressure measurement, especially in the pile-up region.

Numerical and experimental pressure profiles exhibit similar patterns for all the cross-sections and times. Nevertheless, compared to CFD, PIV-based pressure reconstruction yields a generally larger pressure, both in the bulk and in the pile-up (except for $t=6 \mathrm{~ms}$ ). On the one hand, such discrepancies could be attributed to the numerical solution which suffers from a finite resolution in the pile-up region, utilizes a simplified model for the free-surface, neglects compressibility effects, and is implemented upon the experimentally-measured hull motion. On the other hand, inherent uncertainties in PIV analysis are likely to play a significant role 

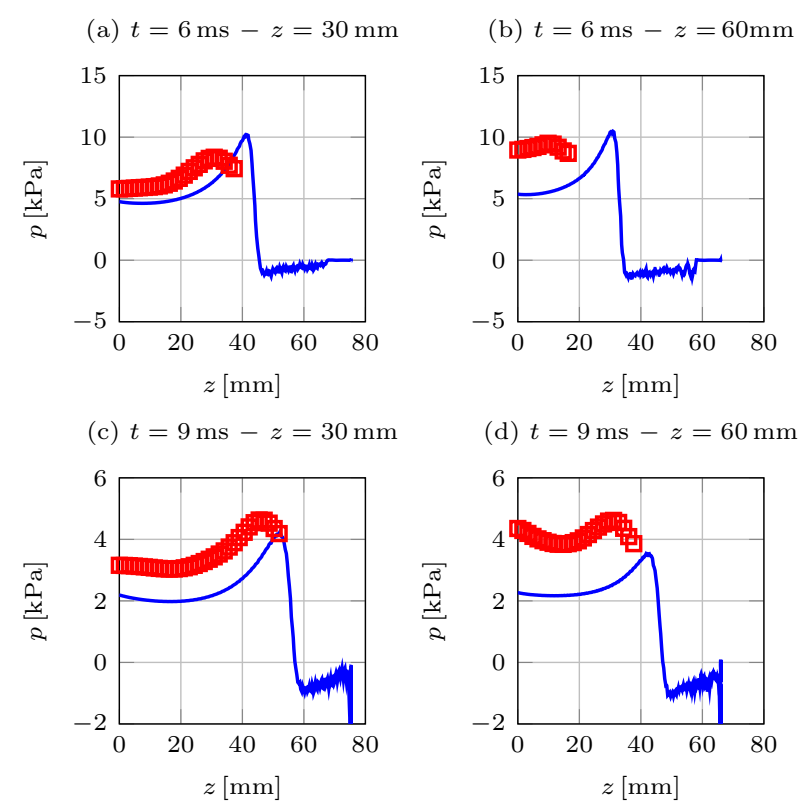

(e) $t=12 \mathrm{~ms}-z=30 \mathrm{~mm}$

(f) $t=12 \mathrm{~ms}$ and $z=60 \mathrm{~mm}$

(g) $t=12 \mathrm{~ms}-z=90 \mathrm{~mm}$
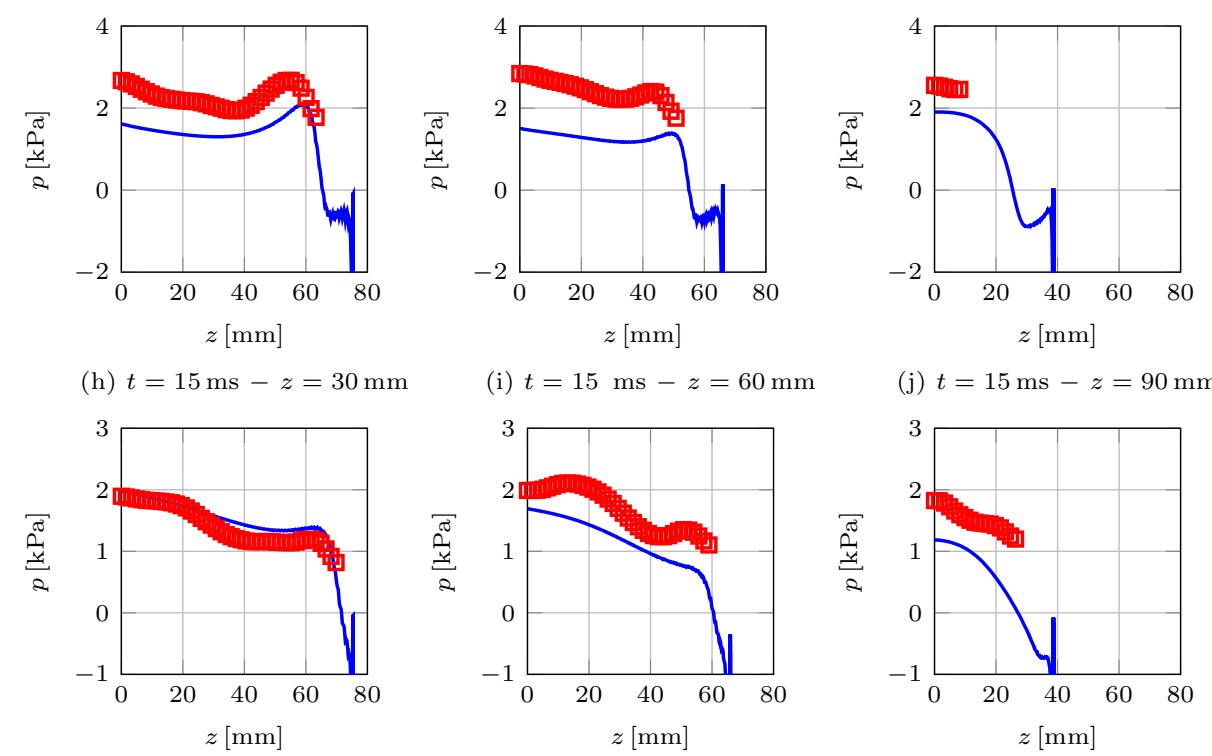

Figure 14: Pressure profiles for different cross-sections and different times after the onset of the impact. (a-j) From left to right: $z=3 \mathrm{~cm}, z=6 \mathrm{~cm}$, and $z=9 \mathrm{~cm}$, and from top to bottom: $t=6 \mathrm{~ms}, t=9 \mathrm{~ms}, t=12 \mathrm{~ms}$, and $t=15 \mathrm{~ms}$. The symmetry plane is at $z=0$. Numerical results are represented with blue continuous lines and experimental findings are plotted using red squares.

on the reconstructed pressure profiles. Particularly important is experimental uncertainties associated with the different repetitions that are executed on the same specimen to scan the 3D space and estimate the pressure everywhere from planar PIV data.

CFD results indicate the formation of negative pressure zones in the water spray, as shown in Figure 14. 
(a) $t=2 \mathrm{~ms}$

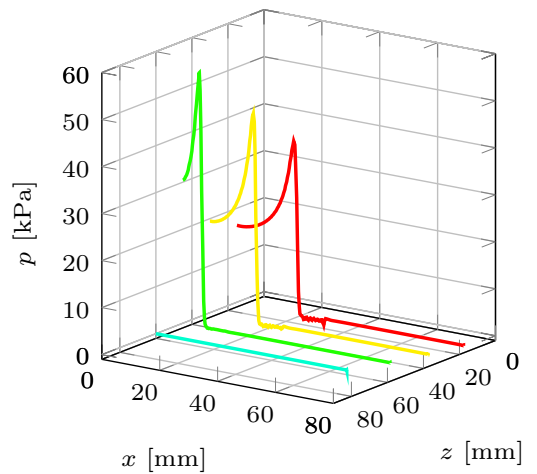

(d) $t=9 \mathrm{~ms}$

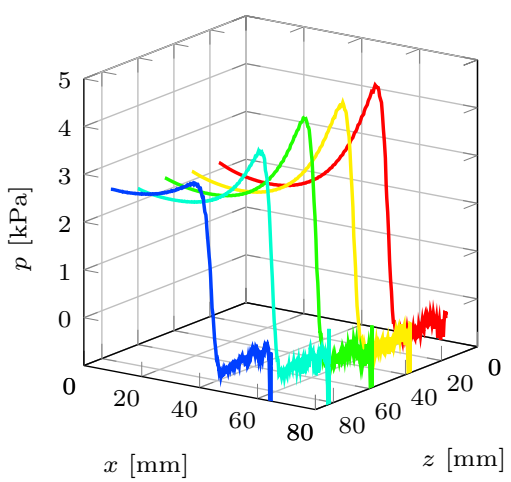

(b) $t=4 \mathrm{~ms}$

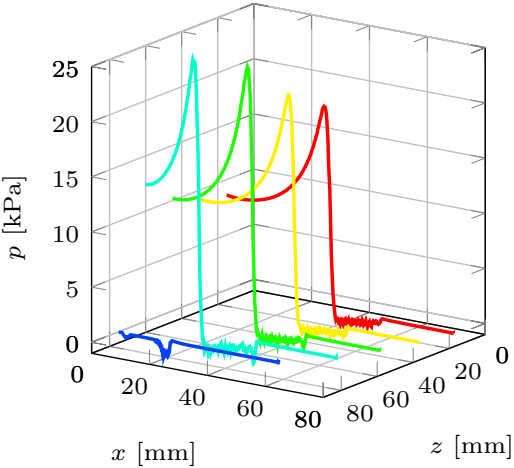

(e) $t=12 \mathrm{~ms}$

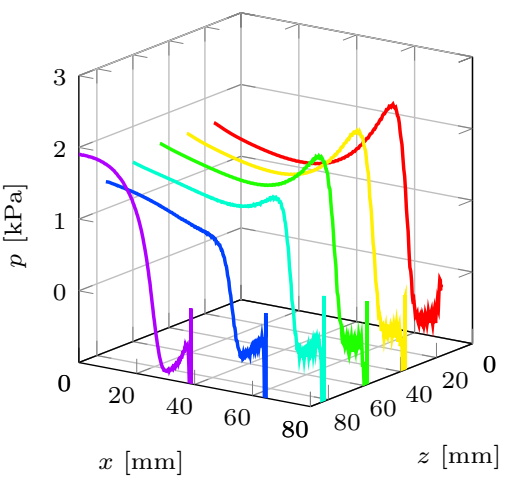

(c) $t=6 \mathrm{~ms}$

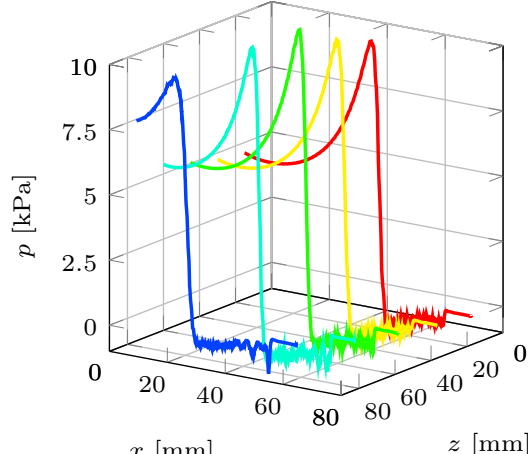

(f) $t=15 \mathrm{~ms}$

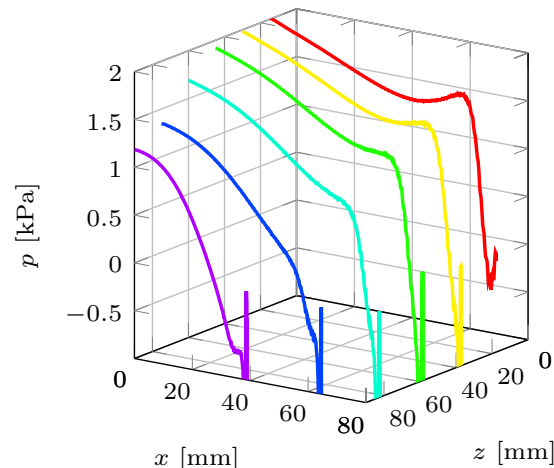

Figure 15: 3D representation of the pressure distribution on the specimen at different times after the onset of the impact: (a) $t=2 \mathrm{~ms}$, (b) $t=4 \mathrm{~ms}$, (c) $t=6 \mathrm{~ms}$, (d) $t=9 \mathrm{~ms}$, (e) $t=12 \mathrm{~ms}$, (f) $t=15 \mathrm{~ms}$. Red lines represent $z=15 \mathrm{~mm}$, yellow line represent $z=30 \mathrm{~mm}$, green line represent $z=45 \mathrm{~mm}$, cyan line represent $z=60 \mathrm{~mm}$, blue line represent $z=75 \mathrm{~mm}$, and purple line represent $z=90 \mathrm{~mm}$

Negative pressure values should be interpreted as below the pressure away from the impact, whereby we set $p=0$ on the top portion of the outer boundary. The formation of these negative pressure regions can be explained by the high speed of the water spray with respect to the bulk flow.

A more comprehensive insight into the hydrodynamic load generated during the water impact is obtained by comparing the 3D depictions of the pressure field in Figure 15 and in Figure 16, with the evolution of the free surface, displayed in Figure 10 and in Figure 11. For $t \leq 9 \mathrm{~ms}$, the hydrodynamic pressure is maximized in the pile-up, in line with theoretical predictions (Mei et al., 1999; Wagner, 1932; Zhao et al., 1996), and numerical simulations (Facci et al., 2015; Seng et al., 2012), on the 2D water impact of wedges, and experimental results on elongated wedges, where 3D effects are negligible (Panciroli and Porfiri, 2013; Panciroli et al., 2015; Zhao et al., 1996). The pressure minimum is not attained at the keel, in agreement with findings in (Facci et al., 2015), for the impact of $2 \mathrm{D}$ wedges with $25^{\circ}<\beta<40^{\circ}$.

Moreover, Figure 15 and 16 demonstrate that for $t \leq 6 \mathrm{~ms}$, the pressure increases moving from the 
(a) $t=2 \mathrm{~ms}$

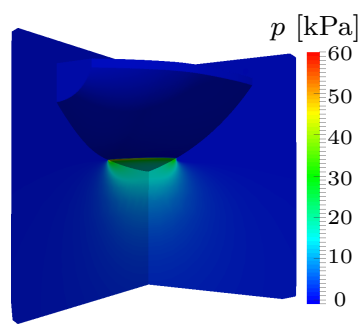

(d) $t=9 \mathrm{~ms}$

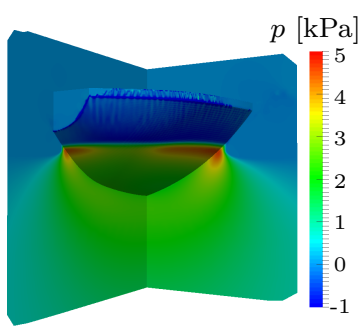

(b) $t=4 \mathrm{~ms}$

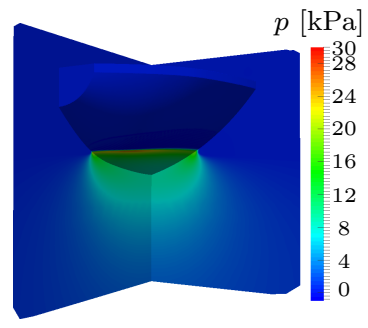

(e) $t=12 \mathrm{~ms}$

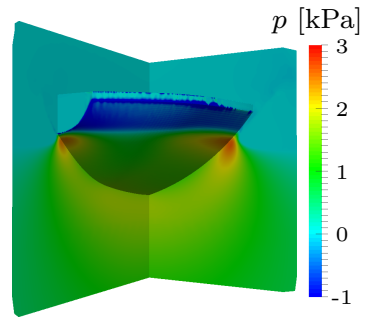

(c) $t=6 \mathrm{~ms}$

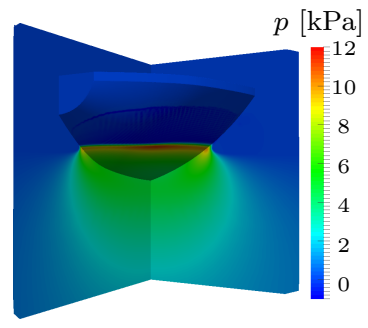

(f) $t=15 \mathrm{~ms}$

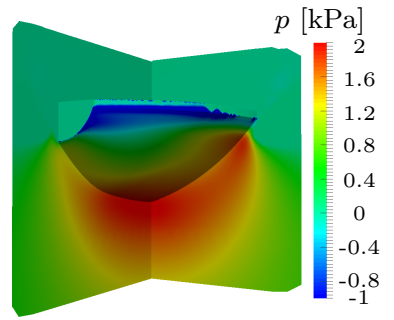

Figure 16: Pressure contour plots on the hull and con the symmetry planes at different time instants: (a) $t=2 \mathrm{~ms}$, (b) $t=4 \mathrm{ms,}$ (c) $t=6 \mathrm{~ms}$, (d) $t=9 \mathrm{~ms}$, (e) $t=12 \mathrm{~ms}$, and (f) $t=15 \mathrm{~ms}$.

Figure 17 shows the maximum hydrodynamic pressure on the impacting hull as a function of time for different cross-sections. For each $x y$-plane, the maximum hydrodynamic loading is attained at the onset of the impact and is gradually reduced as time progresses. As also evidenced in Figures 14, 15, and 16, the maximum pressure is reduced moving from the $z=0$ to the bow.

\subsection{Energy}

Here, we study the energy transfer between the impacting body and the water, utilizing the methodology introduced in (Panciroli et al., 2015), and further developed in (Jalalisendi et al., 2015a). The variation 

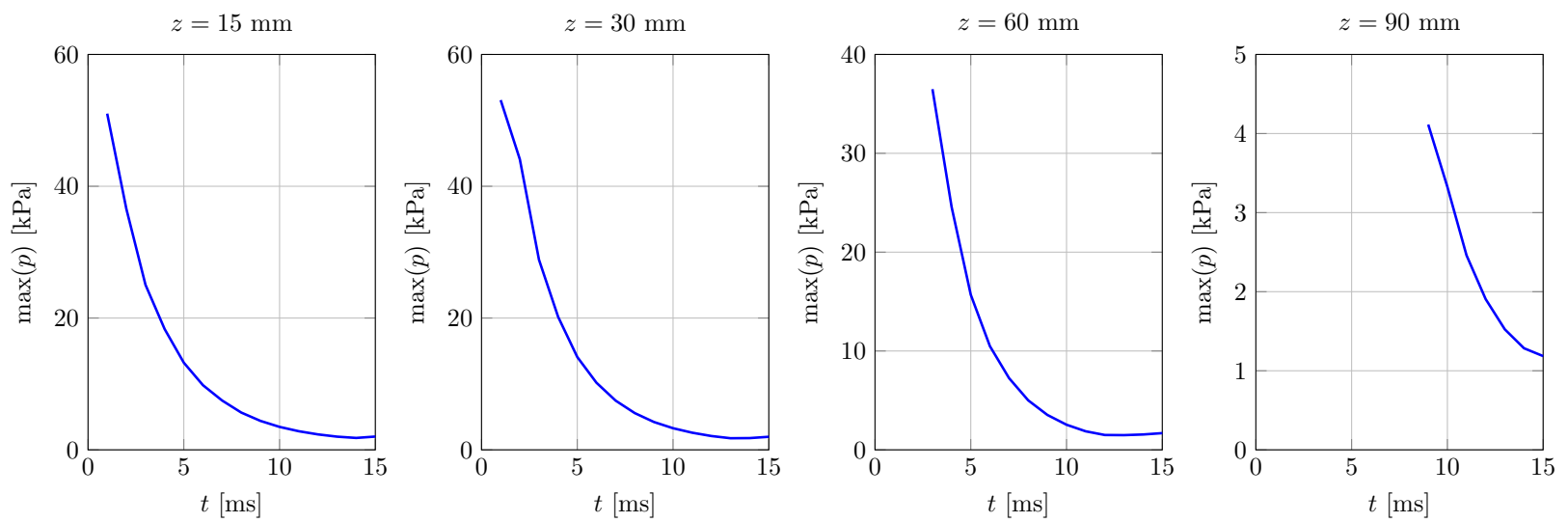

Figure 17: Maximum pressure for different cross-sections as a function of time: (a) $z=15 \mathrm{~mm}$, (b) $z=30 \mathrm{~mm}$, (c) $z=60 \mathrm{~mm}$, and (d) $z=90 \mathrm{~mm}$.

of mechanical energy within the water bulk $\Delta E_{\mathrm{bulk}}(t)=E_{\mathrm{bulk}}(t)-E_{\mathrm{bulk}}(0)$ is reported in Figure 18. In Figure 18(a) $\Delta E_{\text {bulk }}(t)$ is normalized with respect to the variation of mechanical energy of the specimen $\Delta E_{\text {hull }}(t)=1 / 2 M\left(\dot{\xi}(0)^{2}-\dot{\xi}^{2}(t)\right)$, and in Figure 18(b) it is normalized with respect to $E_{\text {hull }}(0)=1 / 2 M \dot{\xi}(0)^{2}$.

Experimental values are retrieved through Eq. (7), and numerical values are computed using Eq. (4). Experimental and numerical results show that most of the mechanical energy (more than $60 \%$ ) that is lost by the falling body is transferred to the risen water. Such a result is in line with the theoretical studies of Scolan and Korobkin (2003), who predict that more than $50 \%$ of the mechanical energy variation of a 3D decelerating body to be imparted to the risen water. Similar results are also presented in (Panciroli et al., 2015) and in (Facci et al., 2015), where the water entry of a 2D wedge is experimentally and numerically studied. Figure 18(a) also suggests that the portion of the energy transferred to the bulk increases with $\xi$, as also proposed in (Jalalisendi et al., 2015a). Differences between computational and experimental results should be attributed to limitations of the PIV analysis for the early stage of the impact (Facci et al., 2015), as well as the accuracy of the computational model in the resolution of the pile-up region. Besides the usual limitations associated with the spatial and temporal discretization, the main limitation of the proposed numerical approach is that the VOF method cannot define a sharp interface between the two phases. Such an approximation is more relevant in the initial stage of the impact, when the dimension of the risen water compares with the grid size, hampering the precise identification of the spray, pile-up, and bulk regions.

\section{Conclusions}

In this paper, we numerically investigated the 3D hydrodynamics generated by the water impact of a solid body, proxying the hull of a marine vessel. The multiphase free-surface flow is modeled through the volume of fluid numerical technique, in which a single set of mass and momentum conservation equations 

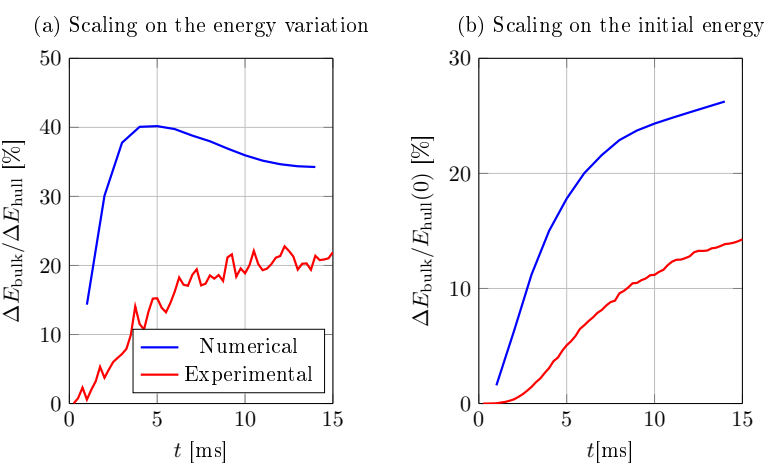

Figure 18: Percentage of the total energy variation of the wedge transferred to the fluid bulk: (a) normalized with respect to the energy variation of the wedge and (b) normalized with respect to the hull mechanical energy at the impact.

are coupled to the evolution of the fluid volume fraction to track the air-water interface. The governing equations are numerically solved through the finite volume technique within an open source computational framework. The study was focused on the water entry of a 3D specimen in free fall, constrained to move only along the vertical direction.

Computational results were compared to $3 \mathrm{D}$ velocity and pressure fields reconstructed from a timeresolved planar PIV system and to the overall hydrodynamic force measured using an accelerometer mounted on the falling body. The numerical simulation was performed by constraining the specimen to follow the experimentally measured trajectory. Numerical and experimental findings on the total hydrodynamic force acting on the hull are within a $15 \%$ difference, except for the very early stage of the impact and toward the end of the experiment.

We also compared numerical and experimental evolutions of the free surface position during the impact, in terms of wetted width and pile-up coefficient as functions of time. Except for the early stage of the impact, CFD and experiments are in very good agreement, both settling down to a pile-up coefficient of 1.25, everywhere on the specimen.. A difference between experimental and theoretical findings is only seen when the specimen is wetted for just a few millimeters of its span, hampering the accurate evaluation of the wetted width from PIV and straining CFD resolution. Interestingly, CFD suggests a monotonic decrease in the pile-up coefficient as a function of time in relation to the 3D nature of the flow, which could cause the specimen to be wetted at a given cross-section due to the pile-up phenomenon at another cross-section. Our planar PIV analysis does not capture this secondary effect, which should be explored using high resolution stereoscopic systems.

Similar to experimental observations, CFD results demonstrate a complex dependence of the water flow on the hull shape. We find that the velocity is maximized in the pile-up region, for all cross-sections and gradually decreases towards the keel. Further, the axial component of the velocity gradually increases along the length of the specimen, as 3D effects become relevant. A strong agreement between CFD and experiments 
is also noted on the hydrodynamic loading experienced by the hull at different cross-sections. Computational results confirm experimental observations on the central role of the hull shape on the pressure profiles, which significantly vary along the length of the hull. From the kinetics and kinematics of the flow, we studied the energy transfer between the impacting body and the water. CFD results and experimental findings both suggest that more than $60 \%$ of the mechanical energy lost by the falling body is transferred to the risen water, and that the portion of the energy transferred to the bulk increases with the hull penetration.

Results of these study are expected to improve our understanding of 3D water entry, by offering a systematic characterization of the flow kinetics and kinematics associated with the impact of a body with multiple curvatures on a quiescent fluid surface. Our computational framework should to aid in the design of marine vessels, by empowering naval engineers with an experimentally-validated open source toolbox to conduct parametric studies on the water entry of ship hulls. Future work will seek to further expand the framework to incorporate fluid-structure interactions during free fall and elucidate 3D hydroelastic phenomena.

\section{Acknowledgement}

This work has been supported by the Office of Naval Research Grant N00014 - 10 - 1 - 0988 with Dr. Y.D.S .Rajapakse as the program manager. Views expressed herein are those of the authors and not of the funding agencies. The authors are grateful to Mr. M. Jalalisendi for his help with the description of the experimental setup from his previous publication with Dr. Porfiri and his assistance with the presentation of the experimental data.

\section{References}

Abrate, S., 2013. Hull slamming. Applied Mechanics Reviews 64, 060803.

Baur, T., Köngeter, J., 1999. PIV with high temporal resolution for the determination of local pressure reductions from coherent turbulence phenomena, in: $3^{\text {rd }}$ International Workshop on PIV'99, Santa Barbara, CA, USA, pp. 101-106.

Carcaterra, A., Ciappi, E., 2004. Hydrodynamic shock of elastic structures impacting on the water: theory and experiments. Journal of Sound and Vibration 271, $411-439$.

Colicchio, G., Greco, M., Faltinsen, O.M., 2006. BEM-level set domain decomposition strategy for nonlinear and fragmented interfacial flows. International Journal for Numerical Methods in Engineering 67, 1385-1419.

Das, K., Batra, R.C., 2011. Local water slamming impact on sandwich composite hulls. Journal of Fluids and Structures 27, $523-551$.

De Rosis, A., Falcucci, G., Porfiri, M., Ubertini, F., Ubertini, S., 2014. Hydroelastic analysis of hull slamming coupling lattice Boltzmann and finite element methods. Computers \& Structures 138, 24-35.

Facci, A.L., Panciroli, R., Ubertini, S., Porfiri, M., 2015. Assessment of PIV-based analysis of water entry problems through synthetic numerical datasets. Journal of Fluids and Structures 55, 484-500.

Faltinsen, O., 1993. Sea loads on ships and offshore structures. volume 1. Cambridge University Press, Cambridge, UK. 
Faltinsen, O.M., Landrini, M., Greco, M., 2004. Slamming in marine applications. Journal of Engineering Mathematics 48, $187-217$

OpenFOAM Foundation, 2013. OpenFOAM user's guide.

Gong, K., Liu, H., Wang, B.l., Kai, G., Hua, L., Ben-long, W., 2009. Water entry of a wedge based on SPH model with an imporved boudary treatment. Journal of Hydrodynamics 21, 750-757.

Hughes, K., Vignjevic, R., Campbell, J., De Vuyst, T., Djordjevic, N., Papagiannis, L., 2013. From aerospace to offshore: Bridging the numerical simulation gaps-Simulation advancements for fluid structure interaction problems. International Journal of Impact Engineering 61, 48-63.

Issa, R.I., 1985. Solution of the implicitly discretized fluid flow equations by operator-splitting. Journal of Computational Physics 62, 40-65.

Jalalisendi, M., Osma, S.J., Porfiri, M., 2015a. Three-dimensional water entry of a solid body: A particle image velocimetry study. Journal of Fluids and Structures 59, 85-102.

Jalalisendi, M., Shams, A., Panciroli, R., Porfiri, M., 2015b. Experimental reconstruction of three-dimensional hydrodynamic loading in water entry problems through particle image velocimetry. Experiments in Fluids 56, 1-17.

Judge, C., Troesch, A., Perlin, M., 2004. Initial water impact of a wedge at vertical and oblique angles. Journal of Engineering Mathematics 48, 279-303.

Khabakhpasheva, T., Korobkin, A., 2013. Elastic wedge impact onto a liquid surface: Wagner's solution and approximate models. Journal of Fluids and Structures 36, 32-49.

Korobkin, A., 1996. Water impact problems in ship hydrodynamics, in: Advances in Marine Hydrodynamics., Computational Mechanics Publications. pp. 323-371.

Korobkin, A., 2004. Analytical models of water impact. European Journal of Applied Mathematics 15, 821-838

Korobkin, A., Malenica, S., 2005. Modified Logvinovich model for hydrodynamic loads on asymmetric contours entering water, in: 20th Int. Workshop on Water Waves and Floating Bodies, Longyearbyen, Norway, May.

Luo, H., Wang, H., Soares, C.G., 2012. Numerical and experimental study of hydrodynamic impact and elastic response of one free-drop wedge with stiffened panels. Ocean Engineering 40, 1-14.

Luo, H., Wang, S., Guedes Soares, C., 2011. Numerical prediction of slamming loads on a rigid wedge subjected to water entry using an explicit finite element method, in: Advances in Marine Structures, Proceedings of the $3^{\text {rd }}$ International Conference on Marine Structures, CRC Press. pp. 41-48.

Maki, K.J., Lee, D., Troesch, A.W., Vlahopoulos, N., 2011. Hydroelastic impact of a wedge-shaped body. Ocean Engineering $38,621-629$

McCue, L.S., 2012. Statistics of extreme slam responses and implications for small craft design. Journal of Ship Production and Design 2, 112-127.

Mei, X., Liu, Y., Yue, D.K., 1999. On the water impact of general two-dimensional sections. Applied Ocean Research 21, 1-15.

Nila, A., Vanlanduit, S., Vepa, S., Van Paepegem, W., 2013. A PIV-based method for estimating slamming loads during water entry of rigid bodies. Measurement Science and Technology 24, 045303.

Oger, G., Doring, M., Alessandrini, B., Ferrant, P., 2006. Two-dimensional SPH simulations of wedge water entries. Journal of Computational Physics 213, 803-822.

OpenFOAM, 2015. www.openfoam.com.

Panciroli, R., Abrate, S., Minak, G., Zucchelli, A., 2012. Hydroelasticity in water-entry problems: Comparison between experimental and SPH results. Composite Structures 94, 532-539.

Panciroli, R., Porfiri, M., 2013. Evaluation of the pressure field on a rigid body entering a quiescent fluid through particle image velocimetry. Experiments in Fluids 54, 1630.

Panciroli, R., Porfiri, M., 2014. Hydroelastic impact of piezoelectric structures. International Journal of Impact Engineering 
66, 18-27.

Panciroli, R., Porfiri, M., 2015. Analysis of hydroelastic slamming through particle image velocimetry. Journal of Sound and Vibration $347,63-78$

Panciroli, R., Shams, A., Porfiri, M., 2015. Experiments on the water entry of curved wedges: High speed imaging and particle image velocimetry. Ocean Engineering 94, 213-222.

Patankar, S., 1980. Numerical Heat Transfer and Fluid Flow. Series in computational methods in mechanics and thermal sciences, McGraw Hill Book Company, New York, USA.

Peterson, R., Wyman, D., Frank, C., 1997. Drop tests to support water-impact and planing boat dynamics theory. Technical Report CSS/TR-97/25. DTIC Document, Naval Surface Warfare Center Panama City, FL, USA.

Raffel, M., Willert, C., Kompenhans, J., 1998. Particle image velocimetry: a practical guide. Springer-Verlag, Berlin Heidelberg, Germany.

Rider, W.J., Kothe, D.B., 1998. Reconstructing volume tracking. Journal of Computational Physics $141,112-152$.

Rusche, H., 2003. Computational fluid dynamics of dispersed two-phase flows at high phase fractions. Ph.D. thesis. Imperial College London (University of London).

Scardovelli, R., Zaleski, S., 1999. Direct numerical simulation of free-surface and interfacial flow. Annual Review of Fluid Mechanics 31, 567-603.

Scolan, Y.M., Korobkin, A.A., 2003. Energy distribution from vertical impact of a three-dimensional solid body onto the flat free surface of an ideal fluid. Journal of Fluids and Structures 17, 275-286.

Seddon, C., Moatamedi, M., 2006. Review of water entry with applications to aerospace structures. International Journal of Impact Engineering 32, 1045-1067.

Semenov, Y.A., Iafrati, A., 2006. On the nonlinear water entry problem of asymmetric wedges. Journal of Fluid Mechanics $547,231-256$.

Seng, S., Jensen, J.J., Pedersen, P.T., 2012. Numerical prediction of slamming loads. Proceedings of the Institution of Mechanical Engineers, Part M: Journal of Engineering for the Maritime Environment 226, $120-134$.

Shams, A., Jalalisendi, M., Porfiri, M., 2015. Experiments on the water entry of asymmetric wedges using particle image velocimetry. Physics of Fluids 27, 027103.

Shams, A., Porfiri, M., 2015. Treatment of hydroelastic impact of flexible wedges. Journal of Fluids and Structures 57, 229-246. Shao, S., 2009. Incompressible SPH simulation of water entry of a free-falling object. International Journal for Numerical Methods in Fluids 59, 91-115.

Stenius, I., Rosén, A., Kuttenkeuler, J., 2006. Explicit FE-modelling of fluid-structure interaction in hull-water impacts. International Shipbuilding Progress 53, 103-121.

Sun, H., Faltinsen, O.M., 2006. Water impact of horizontal circular cylinders and cylindrical shells. Applied Ocean Research $28,299-311$.

Thielicke, W., Stamhuis, E.J., 2014a. PIVlab - Time-Resolved Digital Particle Image Velocimetry Tool for MATLAB (version: $1.32)$.

Thielicke, W., Stamhuis, E.J., 2014b. PIVlab - Towards user-friendly, affordable and accurate digital particle image velocimetry in MATLAB. Journal of Open Research Software 2, e30.

Tveitnes, T., Fairlie-Clarke, A., Varyani, K., 2008. An experimental investigation into the constant velocity water entry of wedge-shaped sections. Ocean Engineering 35, 1463-1478.

Van Nuffel, D., Vepa, K., De Baere, I., Degrieck, J., De Rouck, J., Van Paepegem, W., 2013. Study on the parameters influencing the accuracy and reproducibility of dynamic pressure measurements at the surface of a rigid body during water impact. Experimental Mechanics 53, 131-144.

Van Nuffel, D., Vepa, K., De Baere, I., Lava, P., Kersemans, M., Degrieck, J., De Rouck, J., Van Paepegem, W., 2014. A 
comparison between the experimental and theoretical impact pressures acting on a horizontal quasi-rigid cylinder during vertical water entry. Ocean Engineering 77, 42-54.

Wagner, H., 1932. Über stoß- und gleitvorgänge an der oberfläche von flüssigkeiten. ZAMM - Zeitschrift für Angewandte Mathematik und Mechanik 12, 193-215.

Wang, S., Luo, H., Guedes Soares, C., 2012. Explicit FE simulation of slamming load on rigid wedge with various deadrise angles during water entry, in: Maritime Engineering and Technology, Proceedings of MARTECH 2011, $1^{\text {st }}$ International Conference on Maritime Technology and Engineering, CRC Press. pp. 399-406.

Wang, S., Soares, C.G., 2013a. Numerical study on hydroelastic water entry of a wedge, in: Developments in Maritime Transportation and Exploitation of Sea Resources, Proceedings of IMAM 2013, $15^{\text {th }}$ International Congress of the International Maritime Association of the Mediterranean, CRC Press. pp. 199-208.

Wang, S., Soares, C.G., 2013b. Slam induced loads on bow-flared sections with various roll angles. Ocean Engineering 67 , $45-57$.

Wang, S., Soares, C.G., 2014. Numerical study on the water impact of 3D bodies by an explicit finite element method. Ocean Engineering 78, 73-88.

Wu, G., Sun, H., He, Y., 2004. Numerical simulation and experimental study of water entry of a wedge in free fall motion. Journal of Fluids and Structures 19, 277-289.

Yang, Q., Jones, V., McCue, L., 2012. Free-surface flow interactions with deformable structures using an SPH-FEM model. Ocean Engineering 55, 136-147.

Zarghami, A., Falcucci, G., Jannelli, E., Succi, S., Porfiri, M., Ubertini, S., 2014. Lattice Boltzmann modeling of water entry problems. International Journal of Modern Physics C 25, 1441012.

Zhao, R., Faltinsen, O., Aarsnes, J., 1996. Water entry of arbitrary two-dimensional sections with and without flow separation, in: Proceedings of $21^{\text {st }}$ Symposium on Naval Hydrodynamics, The National Academies Press. pp. 408-423. 